Commentary

\title{
Surprising sirtuin crosstalk in the heart
}

\section{Thaddeus T. Schug and Xiaoling Li}

\author{
Laboratory of Signal Transduction, National Institute of Environmental Health Sciences, National Institutes of \\ Health, Research Triangle Park, NC 27709, USA
}

Running title: Sirtuins benefit the heart

Key words: Sirtuins, insulin-like growth factor, cardiac hypertrophy

Abbreviations and Acronyms: IGF-1: insulin growth factor 1; SIRT1-7: Sirtuins 1-7; Tg: transgenic; MYH7: fetal $\alpha$-myosin heavy chain 7; Foxo: forkhead box transcription factor; ROS: reactive oxygen species; MT-2: metallothionein-2; UCP1: uncoupling protein 1; PI3K: phosphoinositide 3-kinase; AKT: protein kinase B; SGK1: Serum-and Glucocorticoid-regulated Serine/threonine-protein kinase; MAPK: Mitogen-activated protein kinase: ERK: extracellular signal-regulated kinase Correspondence: Thaddeus T. Schug, PhD, Laboratory of Signal Transduction, National Institute of Environmental Health Sciences, National Institutes of Health, Research Triangle Park, NC 27709, USA

Received: 03/12/10; accepted: 03/16/10; published on line: 03/17/10

E-mail: schugt@niehs.nih.gov

Copyright: (C) Schug and Li. This is an open-access article distributed under the terms of the Creative Commons Attribution License, which permits unrestricted use, distribution, and reproduction in any medium, provided the original author and source are credited

Cardiovascular disease is the leading cause of death in the United States, accounting for nearly one third of all mortalities [9]. While lifestyle modifications, drug development, and surgical technologies have increased the average life expectancy of humans, the risk and rates of heart disease continue to grow as the population ages. The process of aging causes unique physiological, histological, and biochemical changes in cardiac tissue. For example, aging cardiac myocytes are incapable of proliferation and cannot be reprogrammed transcriptionally in response to changes in workload [7]. Ageassociated conditions such as buildup of reactive oxygen species, mechanical dysfunction, or other forms of trauma, have been linked to the development of hypertrophy and other cardiac pathologies [6, 14]. Therefore, development of drugs that target agedependent signaling pathways may provide promising therapeutic strategies for the treatment of heart disease.

mIGF-1 protects the heart through crosstalk with SIRT1

A study by Vinciguerra et al. published in the January 2010 issue of Aging furnishes new and important information about the cellular mechanisms leading to the pathogenesis of heart failure [22]. They propose a cardio-protective link between locally acting insulinlike growth factor (mIGF-1) and the NAD+-dependent deacetylase SIRT1. Typically, it is thought that the highly conserved IGF-1 and sirtuin signaling pathways play antagonizing roles in mammalian physiology. IGF1 acts primarily as a growth hormone and signaling factor. Mice lacking GH/IGF-I signaling and IGF-1 receptor heterozygous knockout mice have longer lifespans, and overexpression of a hormone known to inhibit insulin/IGF-1 signaling extends lifespan [5, 18]. However, the complex structure of the Igf- 1 gene gives rise to multiple peptide isoforms that have contrasting functions [21]. Notably, the mIGF-1 isoform, which is expressed at high levels in neonatal tissues and adult liver, promotes regenerative properties in damaged heart tissue [21].

SIRT1, the mammalian orthologue of yeast Sir2, is a highly conserved NAD+-dependant protein deacetylases that has emerged as an important regulator of aging and metabolic disease [2]. SIRT1 and its family members are reported to promote longevity in different model organisms, including yeast, worm and fly $[2,13]$. The mammalian SIRT1 protein is primarily nuclear, and its functions have been tied to metabolism, cell survival and stress response [4]. The full-body SIRT1 knockout 
mouse displays ventricular adult heart abnormalities [10], but a severe developmental phenotype, together with high neonatal mortality rates make use of it difficult to study the physiological role of SIRT1 in the adult heart. Interestingly, high levels of SIRT1 expression ( $>9$-fold) in the heart causes hypertrophy, loss of cardiac function, and elevated apoptosis [1]. On the other hand, moderate overexpression (2.5 to 7-fold) of SIRT1 in transgenic mouse hearts protects against oxidative stress, and results in increased expression of antioxidants [1]. As well, SIRT1 expression is increased in the hypertrophic heart of rodents and monkeys, though its functional relevance remains unclear [19].

Vinciguerra et al. hypothesize that although SIRT1 and circulating IGF-1 play opposite roles, the local mIGF-1 isoform displays a novel cross-talk signaling program with SIRT1, which results in cardiomyocite protection from hypertrophic and oxidative stress. They show that mIGF-1-mediated activation of SIRT1 induces expression of the protective signaling molecules UCP1, adiponectin, and MT2 [22]. They suggest that SIRT1 activation in the heart may also elicit protection from hypertrophy by restoring expression of fetal $\alpha$-myosin heavy chain 7 . The authors note an important distinction between circulating IGF-1 and mIGF-1. Typically, circulating IGF-1 activates PI3K/AKT/mTor and MAP kinase pathways, whereas mIGF-1 signals through PDK1 and SGK1 [22]. They conclude that the divergent signaling mechanisms between the two IGF-1 isoforms may account for their opposing effects in heart tissue (Figure 1).

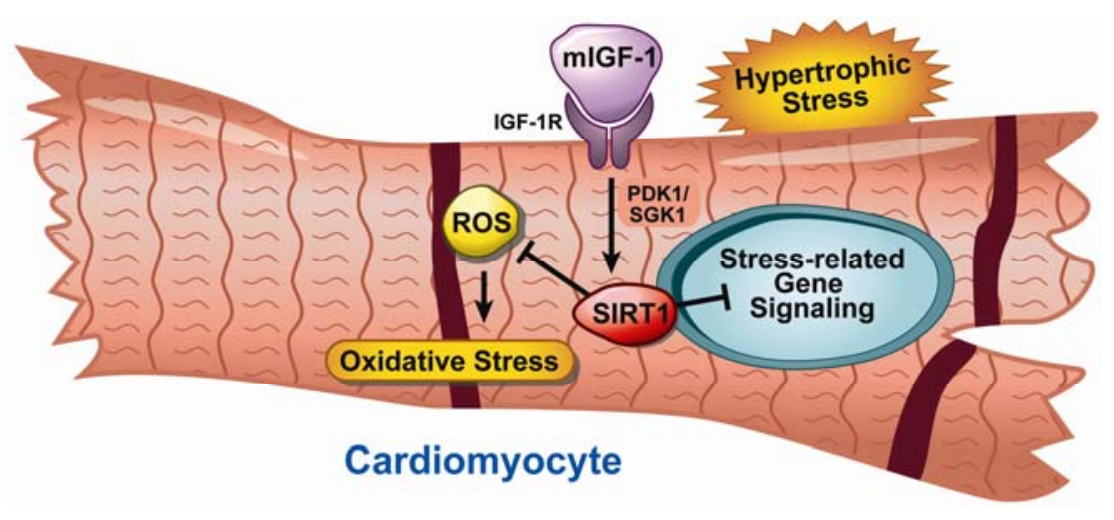

Figure 1. Model depicting the role of mIGF-1 regulation in cardiomyocytes.

Table 1: Mammalian Sirtuin cardiac phenotypes

\begin{tabular}{|c|c|c|c|}
\hline Sirtuin & Location & Activity & Transgenic Mouse Phenotype \\
\hline \multirow{3}{*}{ SIRT1 } & \multirow{3}{*}{ Nucleus } & \multirow{3}{*}{ NAD-dependent deacetylase } & KO - Ventricular abnormalities \\
\hline & & & Tg - Overexpression (>9-fold) - Cardiac hypertrophy \\
\hline & & & $\begin{array}{r}\text { Tg - Overexpression (2.5 to 7-fold) - Protection from oxidative stress } \\
\text { - Increased expression of antioxidants }\end{array}$ \\
\hline \multirow{2}{*}{ SIRT 3} & \multirow{2}{*}{ Mitochondria/nucleus } & \multirow{2}{*}{ NAD-dependent deacetylase } & KO - Cardiac hypertrophy \\
\hline & & & Tg - Overexpression - Cardio-protective \\
\hline SIRT 7 & Not established & $\begin{array}{l}\text { NAD-dependent deacetylase/ } \\
\text { ribosomal biogenesis }\end{array}$ & KO - Cardiac hypertrophy \\
\hline
\end{tabular}




\section{Other sirtuins benefit the heart}

Recently, two additional sirtuin family membersSIRT3 and SIRT7--have been shown to have beneficial functions in the heart (Table 1), [17, 20]. SIRT3, which consists of two isoforms $(\approx 28 \mathrm{kDa}$ and $\approx 44 \mathrm{kDa})$ was initially identified as a mitochondrial protein, but has since been identified in the nucleus as well $[15,16]$. Similar to SIRT1, SIRT3 uses NAD+ as a cofactor for the deacetylation of target substrates [2,12]. Cellular energy status, reflected in NAD+ levels and NAD+/NADH ratios, are thought to influence SIRT1 and SIRT3 expression and activity [8]. For example, mild stress conditions, such as mechanical stress to the heart and calorie restriction (CR), are presumed to reduce NADH levels, thus increasing the NAD+/NADH ratio. These alterations in cellular energy provide fuel to drive induction of SIRT3 during physiologic and mild hypertrophy.

SIRT3-deficient mice show signs of cardiac hypertrophy and interstitial fibrosis at 8 weeks of age, while SIRT3 transgenic overexpressing mice are protected from application of hypertrophic stimuli [17]. SIRT3 blocks the cardiac hypertrophic response through activation of Foxo-dependent antioxidants, manganese superoxide dismutase (MnSOD) and catalase, as well as suppressing ROS-mediated Ras activation and the downstream MAPK/ERK and PI3K/Akt signaling pathways [17]. SIRT1 and SIRT3 appear to share similar ROS-accumulating end-point targets that cause cardiac hypertrophy. Use and development of sirtuinspecific activators and inhibitors may help further dissect the collaborative functions of SIRT1 and SIRT3 in the heart.

Less is known about the physiological role of SIRT7 in the heart. SIRT7 is a nuclear protein that associates with rDNA and interacts with RNA [3]. It is not certain whether SIRT7 exhibits NAD+-dependent deacetylase activity, but reports suggest that it does respond to metabolic conditions by stimulating ribosomal biogenesis in dividing cells [11]. SIRT7-deficient mice develop heart hypertrophy and inflammatory cardiomyopathy, which is characterized by extensive fibrosis [20]. SIRT7 appears to regulate heart cell death and damage by inhibiting p53, Ras, and Akt signaling pathways [20]. The molecular details explaining how SIRT7 targets these pathways remains unclear.

\section{Conclusions}

Sirtuins are longevity factors that also appear to regulate critical cardio-protective pathways in the mammalian heart. To date, three family members-SIRT1, SIRT3, and SIRT7-have been shown to block stress-induced cardiac hypertrophy by impinging upon ROS generation. It is interesting that knockout mice for each sirtuin isotype exhibit heart abnormalities, while transgenic overexpression of all three provides protection from cardiac hypertrophy. More investigation using conditional knockout models and specific activators is needed to elucidate the distinct molecular functions of each sirtuin. These studies will have profound implications, not only for the management of heart failure, but also for other stress-associated diseases.

\section{CONFLICT OF INTERESTS STATEMENT}

The authors of this manuscript have no conflict of interest to declare.

\section{REFERENCES}

1. Alcendor RR, Gao S, Zhai P, Zablocki D, Holle E, Yu X, Tian B, Wagner T, Vatner SF, and Sadoshima J. Sirt1 regulates aging and resistance to oxidative stress in the heart. Circ Res. 2007; 100:1512-1521.

2. Blander G, and Guarente L. The Sir2 family of protein deacetylases. Annu Rev Biochem. 2004; 73:417-435.

3. Ford E, Voit R, Liszt G, Magin C, Grummt I, and Guarente L. Mammalian Sir2 homolog SIRT7 is an activator of RNA polymerase I transcription. Genes Dev. 2006; 20:1075-1080.

4. Haigis, MC and Sinclair DA. Mammalian sirtuins: biological insights and disease relevance. Annu Rev Pathol. 2010; 5:253-95.

5. Holzenberger $M$, Dupont J, Ducos B, Leneuve $P$, Geloen $A$, Even PC, Cervera $P$, and Le Bouc Y. IGF-1 receptor regulates lifespan and resistance to oxidative stress in mice. Nature. 2003; 421:182-187.

6. Hsu CP, Odewale I, Alcendor RR, and Sadoshima J. Sirt1 protects the heart from aging and stress. Biol Chem. 2008; 389:221-231.

7. Levine TB, Levine AB, Bolenbaugh J, and Green PR. Reversal of heart failure remodeling with age. Am J Geriatr Cardiol. 2002; 11:299-304.

8. Lin SJ, Ford E, Haigis M, Liszt G, and Guarente L. Calorie restriction extends yeast life span by lowering the level of NADH. Genes Dev. 2004; 18:12-16.

9. Lloyd-Jones, D, Adams RJ, Brown, TM Carnethon M, Dai S, De Simone G, Ferguson TB, Ford E, Furie K, Gillespie C, Go A, Greenlund K, et al. Executive Summary: Heart Disease and Stroke Statistics--2010 Update: A Report From the American Heart Association. Circulation. 2010; 121:948-954.

10. McBurney MW, Yang X, Jardine K, Hixon M, Boekelheide K, Webb JR, Lansdorp PM, and Lemieux $M$. The mammalian SIR2alpha protein has a role in embryogenesis and gametogenesis. Mol Cell Biol; 2003; 23:38-54.

11. Michishita E, Park JY, Burneskis JM, Barrett JC, and Horikawa I. Evolutionarily conserved and nonconserved cellular localizations and functions of human SIRT proteins. Mol Biol Cell. 2005; 16:4623-35.

12. Opie LH and Owen P. Effects of increased mechanical work by isolated perfused rat heart during production or uptake of 
ketone bodies. Assessment of mitochondrial oxidized to reduced free nicotinamide-adenine dinucleotide ratios and oxaloacetate concentrations. Biochem J. 1975; 148:403-415.

13. Rogina B and Helfand SL. Sir2 mediates longevity in the fly through a pathway related to calorie restriction. Proc Natl Acad Sci U S A. 2004; 101:15998-16003.

14. Rosamon W, Flegal K, Friday G, Furie K, Go A, Greenlund K, Haase N, Ho M, Howard V, Kissela B, Kittner S, Lloyd-Jones D, McDermott $M$, et al. Heart disease and stroke statistics--2007 update: a report from the American Heart Association Statistics Committee and Stroke Statistics Subcommittee. Circulation. 2007; 115:e69-171.

15. Scher MB, Vaquero A, and Reinberg D. SirT3 is a nuclear NAD+-dependent histone deacetylase that translocates to the mitochondria upon cellular stress. Genes Dev. 2007; 21:920-928. 16. Schwer B, North BJ, Frye RA, Ott M, and Verdin E. The human silent information regulator (Sir)2 homologue hSIRT3 is a mitochondrial nicotinamide adenine dinucleotide-dependent deacetylase. J Cell Biol. 2002; 158:647-657.

17. Sundaresan, NR, Gupta M, Kim G, Rajamohan SB, Isbatan A, and Gupta MP. Sirt3 blocks the cardiac hypertrophic response by augmenting Foxo3a-dependent antioxidant defense mechanisms in mice. J Clin Invest. 2009; 119:2758-2771.

18. Tatar M, Bartke A, and Antebi A. The endocrine regulation of aging by insulin-like signals. Science. 2003; 299:1346-51.

19. Vahtola $E$, Louhelainen $M$, Merasto $S$, Martonen E, Penttinen $\mathrm{S}$, Aahos I, Kyto V, Virtanen I, and Mervaala E. Forkhead class O transcription factor 3a activation and Sirtuin1 overexpression in the hypertrophied myocardium of the diabetic Goto-Kakizaki rat. J Hypertens. 2008; 26:334-344.

20. Vakhrusheva O, Smolka C, Gajawada P, Kostin S, Boettger T, Kubin T, Braun T, and Bober E. Sirt7 increases stress resistance of cardiomyocytes and prevents apoptosis and inflammatory cardiomyopathy in mice. Circ Res. 2008; 102:703-710.

21. Winn N, Paul A, Musaro A, and Rosenthal N. Insulin-like growth factor isoforms in skeletal muscle aging, regeneration, and disease. Cold Spring Harb Symp Quant Biol. 2002; 67:507518.

22. Vinciguerra, $M$, Santini MP, Claycomb WC, Ladurner AG, Rosenthal N. Local IGF-1 isoform protects cardiomyocytes from hypertrophic and oxidative stress via SirT1 activity. Aging. 2010; 2: 43-62. 